\title{
Control and Simulation of Temperature for Injection Molding Machines Based on PID Neural Networks
}

\author{
Shaojia $\mathrm{He}^{\mathrm{a}}$, Lvguang Shi ${ }^{\mathrm{b}}$ \\ School of Mechanical and Electrical Engineering, Guilin University of Electronic Technology, Guilin \\ 541004, Guangxi, China \\ ahmhsj@sina.com, bslgyjj@sina.com
}

Keywords: PID, Neural Networks, Simulation, Control.

\begin{abstract}
Focused on the dynamic and static performances of a nonlinear time-variant system with large delay-time, and the shortages of great control deviation and slow rate of regulation in the traditional PID control systems for injection molding machines, this paper has established injection molding machine temperature controller model with using PID neural networks, completed the simulation and the contrast to the traditional PID control simulation for using MATLAB software. Simulation results demonstrate that the PID neural networks controller has strong ability to adapt working conditions and inhibit interference, and better than conventional PID controller to control the temperature.
\end{abstract}

\section{Introduction}

In the injection molding process, the melt temperature which depends on the temperature of the friction between the barrel and the screw is an important parameter. If the temperature of barrel is too low, the plastic between the screw and barrel will have a greater shear force, it will damage the machine. If the temperature is too high, the plastic molecules will break down, the plastic will appear foaming phenomenon, which is generally not allowed to happen, especially in precision injection molding. ${ }^{1]}$ In this paper, the controlled object is the barrel heaters of injection molding machine, its purpose is to control the temperature. In industrial process control, the traditional PID control technology plays an important role in the temperature control system, but the parameters of traditional PID controller requires accurate mathematical model of the object to be adjusted, through complex calculations, its 3 parameters in the control system operation are generally fixed, and difficult to online adjustment. In the process of temperature control, the barrel of the injection molding machine is a nonlinear time-variant system with large delay-time and difficult to obtain an accurate mathematical model, so the traditional PID control is difficult to achieve optimal results in the injection molding machine temperature control. The PID neural networks is a intellective-control technology by combining PID control rule with neural network. It has the advantages of simple structure, clear physical meanings of parameters, as well as the functions of nonlinear reflection ability and self-learning and self-adaptive of the neural networks. It does not depend on the precise mathematical model of controlled object, which is particularly suitable for the nonlinear time-variant complex systems with large delay-time ${ }^{[2]}$. In view of this, this study will use the PID neural networks to control the barrel temperature and the simulation of PID neural networks control and conventional PID control to analyze and compare the effect of both control by MATLAB software.

\section{PID neural networks controller model}

\subsection{Brief introduction}

The single input-output system of PID neural networks control model shown in Figure 1, a simple three-tier structure $2 \times 3 \times 1$, comprising two input layer neurons, three hidden layer neurons and one output layer neurons. Two neurons in the input layer, one as a given variable $r$, another for output variable y; 3 hidden layer neurons, which are proportional function, integral function, differential function; one element of the output layer is the nerve is the control variable that output objects needed. 
Form of PID neural networks is a multilayer forward neural network, the number of its layers of neurons, connection, connection weights are based on the fundamental principles and past experience of the PID control rules, and the purpose is to ensure that the system stable and fast convergence in the initial state.

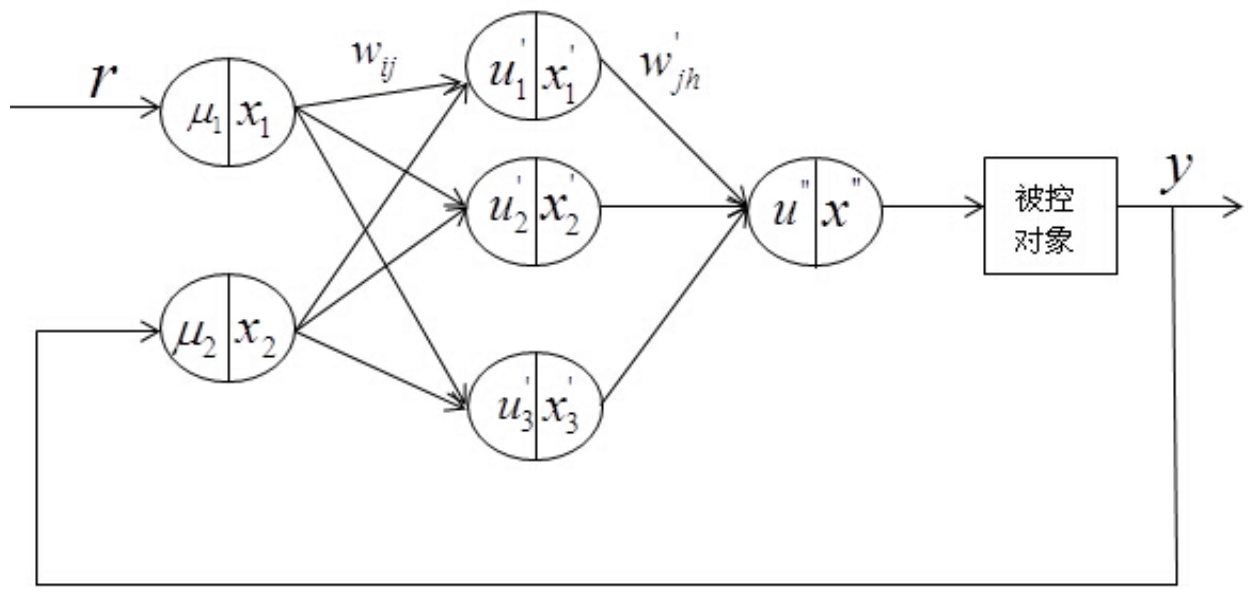

Fig.1 PIDNN single-variable control system architecture

\subsection{The forward algorithms}

Any sampling time $\mathrm{k}$, the formula which is applied to single input-output system of PID neural networks is as follows:

2.2.1 The input layer

The input layer is composed of two identical internal structure of neurons, input-output relationship is as follows:

$x_{\mathrm{i}}(k)=\mu_{i}(k)$

2.2.2 The hidden layer

The hidden layer are composed of internal structure of three different neurons which are defined as proportional element, integral element, differential element, the same input value is calculated as:

$\mu_{j}^{\prime}(k)=\sum_{i=1}^{2} w_{i j} x_{i}(k)$

Proportional element input-output functions:

$x_{1}^{\prime}(k)=\mu_{1}^{\prime}(k)$

Integral element input-output functions:

$x_{2}^{\prime}(k)=x_{2}^{\prime}(k-1)+\mu_{2}^{\prime}(k)$

Differential element input-output functions:

$x_{3}^{\prime}(k)=u_{3}^{\prime}(k)-\mu_{3}^{\prime}(k-1)$

2.2.3 The output layer

The output layer has one neuron, the input of output layer is a weighted sum of all output values of the hidden layer neurons. Calculated as follows:

$\mu_{h}{ }_{h}(k)=\sum_{j=1}^{3} w_{j h}^{\prime} x_{j}^{\prime}(k)$

Input-output relationship between the output layer neurons is as follows:

$x_{h}{ }_{h}(k)=\mu^{\prime \prime}{ }_{h}(k)$

\subsection{The back propagation algorithm}

The update of connection weights in PID neural network are obtained through the error back propagation learning algorithm. The purpose is to make the mean of sum of squared errors between the desired output and the actual output reaches the minimum. Calculated as follows: 


$$
J=\frac{1}{n} \sum_{k=1}^{n}[r(k)-y(k)]^{2}=\frac{1}{n} \sum_{k=1}^{n} e^{2}(k)
$$

In the formula (8): $\mathrm{n}$ is the number of samples of each batch, $\mathrm{k}$ is the sampling points. The weights through training and leaning of $\mathrm{n}$ steps are determined by follow formulas.

2.3.1 The updated of connection weights for the hidden layer to the output layer

$$
w_{j h}^{\prime}\left(n_{o}+1\right)=w_{j h}^{\prime}\left(n_{o}\right)+\eta_{j h}^{\prime} \frac{\partial J}{\partial w_{j h}^{\prime}}
$$

In the formula (9):

$$
\frac{\partial J}{\partial w_{j h}^{\prime}}=\frac{\partial J}{\partial y} \frac{\partial y}{\partial x_{h}^{\prime \prime}} \frac{\partial x_{h}^{\prime \prime}}{\partial \mu_{h}^{\prime \prime}} \frac{\partial \mu_{h}^{\prime \prime}}{\partial w_{j h}^{\prime}}=-\frac{1}{n} \sum_{i=1}^{n}[r(k+1)-y(k+1)] \cdot \operatorname{sgn}\left[\frac{y(k+1)-y(k)}{x_{h}^{\prime \prime}(k)-x_{h}^{\prime \prime}(k-1)}\right] \cdot x_{j}^{\prime}(k)
$$

In the formula: $\mathrm{j}(=1,2,3)$ is a neural network hidden layer number, $\mathrm{h}(=1)$ is the number of output layer neurons. In the formula (9), the partial differential of controlled object for the output to the input is calculated by the symbolic functions of relative transformation.

2.3.2 The updated of connection weights for the input layer to the hidden layer

$$
w_{i j}\left(n_{o}+1\right)=w_{i j}\left(n_{o}+1\right)+\eta_{i j} \frac{\partial J}{\partial w_{i j}}
$$

In the formula (10):

$$
\begin{aligned}
& \frac{\partial J}{\partial w_{i j}}=\frac{1}{n} \sum_{i=1}^{n}[r(k+1)-y(k+1)] \cdot \operatorname{sgn}\left[\frac{y(k+1)-y(k)}{x_{h}^{\prime \prime}(k)-x_{h}^{\prime \prime}(k-1)}\right] \cdot w_{j h}^{\prime}\left(n_{o}\right) \\
& \cdot \operatorname{sgn}\left[\frac{x^{\prime}(k)-x^{\prime}(k-1)}{u_{j}^{\prime}(k)-u_{j}^{\prime}(k-1)}\right] \cdot x_{i}(k) \cdot \operatorname{sgn}\left[\frac{x^{\prime}(k)-x^{\prime}(k-1)}{u_{j}^{\prime}(k)-u_{j}^{\prime}(k-1)}\right] \cdot x_{i}(k)
\end{aligned}
$$

In the formula: $\mathrm{i}(=1,2)$ is the number of neurons in the input layer, $\mathrm{j}(=1,2,3)$ is the number of hidden layer neuron network.

\section{The barrel temperature control system}

Barrel temperature refers to the heating temperature of the cylinder surface is the only source of external heating plasticizing unit. Barrel is be heated by the heating coil, in the process cartridge can be heated in three parts based on the plasticizing mechanism in the material cylinder, each segment is equipped with a group of electric wire, and is mounted a thermocouple in the geometric center to detect the temperature of each section ${ }^{[3]}$, the first section of barrel is a solid transportation section, the temperature should be slightly lower, but the feed opening must be cooled to prevent "bridging" phenomenon caused overheating, which will not make feeding smooth. The second is the compression section, the temperature should be 20 to 25 degrees higher than first section. The third is a metering section, the general temperature of 0 to 25 degrees higher than the second paragraph to ensure that temperature gradient of the material in the molten state and feeding between tube surface temperature and tube wall temperature exists, and close to the barrel wall temperature of the melt temperature and the barrel wall temperature is close to the melt temperature. Each section is mainly affected by the temperature of the heating power of control this section, but other segments have some affection to each other. Because of the general use of the barrel constant temperature control, and the small temperature difference, the impact can be seen as a constant, the three associated section were treated as independent single input-output links to control. Under certain conditions of the screw back pressure and rotational speed, barrel temperature and the melt temperature is approximately proportional to change, and the melt temperature directly affects the flow properties and viscosity of the melt. So the stability of cylinder temperature plays an important role on the thermostatic control of melt temperature ${ }^{[4]}$. 
The study of temperature characteristics of the barrel is to establish an accurate, appropriate and practical mathematical model control system. The mathematical model of barrel heating system could be determined using response curve. With ignoring to the coupling of adjacent segments and model order reduction of step response curve obtained, we know that the barrel of the injection molding machine heating zone models have a first-order response characteristics, and the study can be approximated to first-order inertia links with delay-time to approximate the dynamic relationship between the output and input process ${ }^{[5]}$, the transfer function $[\mathrm{G}(\mathrm{s})]$ as follows:

$$
G(s)=\frac{t(s)}{u(s)}=\frac{K e^{-\tau s}}{1+T s}
$$

In the formula (11): $t(s)$ is the temperature variable, $u(s)$ is the variable control voltage, $K$ is the amplification factor, $\mathrm{T}$ is the inertia time constant, $\mathrm{S}$ is the Laplace transform factor, and $\tau$ is the lag time. Through the reaction curve, finally obtained parameter values: $K=150, T=1000, \tau=50$, completed the establishment of the mathematical model of the barrel of the heating segment.

\section{Temperature Control Simulation}

In this paper simulation of unit step response and Anti-interference response were carried out in MATLAB environment.

\subsection{Unit step response experiments}

The initial state of connection weights of PID neural networks can be equivalent to the PID controller $^{[2]}$. So the initial value of connection weights for the hidden layer to the output layer are as follows: $w_{11}^{\prime}=K_{P}, w_{21}^{\prime}=K_{i}, w_{31}^{\prime}=K_{d}$. The initial connection weights of the input layer to the hidden layer are as follows: $w_{1 j}=1, w_{2 j}=-1(\mathrm{j}=1,2,3)$. The learning rate $: \eta=0.01$. Through online learning and adjusting, each of the connection weights of PID neural network controller obtained the following results: $w_{11}=0.9321, w_{21}=-0.3022, w_{12}=0.3001, w_{22}=-0.3001, w_{13}=0.2023, w_{23}=$ $-0.1989, w_{11}^{\prime}=0.7032, w_{21}^{\prime}=0.0451, w_{31}^{\prime}=0.2999$.

Unit step response simulation results are shown in Figure2 and Figure3 (horizontal unit of the figures: seconds). The unit step response curve of conventional PID control is shown in Figure 2 and the unit step response curve of PID neural networks control is shown in Figure 3. Through the comparison, the neural network PID control has a small overshoot of only $3.52 \%$ and rapids response, which take only 60 seconds to reach error bands of 5\%, and the effect of control is better than conventional PID control.

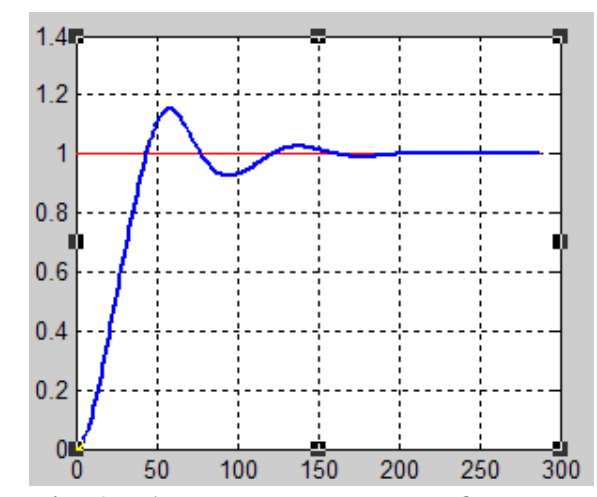

Fig.2 The step response of PID

\subsection{The Anti-interference experiments}

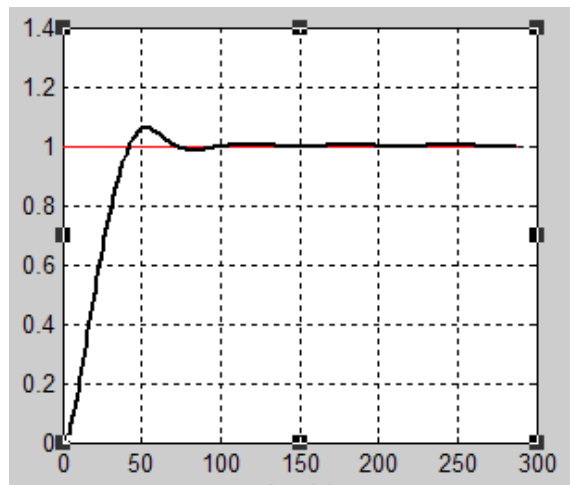

Fig.3 The step response of PIDNN

Adding with an amplitude of 0.2 of step disturbance between 170-175 seconds to Controlled object, it can be obtained the test response curves shown in in figure4 and figure5. From figure4 and figure5, the PID neural networks control reflects the great advantages, which can quickly suppress the disturbance with small temperature fluctuations and relatively short time of adjustment when 
perturbations occurs, and on the other hand, the conventional PID control will cause the occurrence of large fluctuations and vibration in temperature.

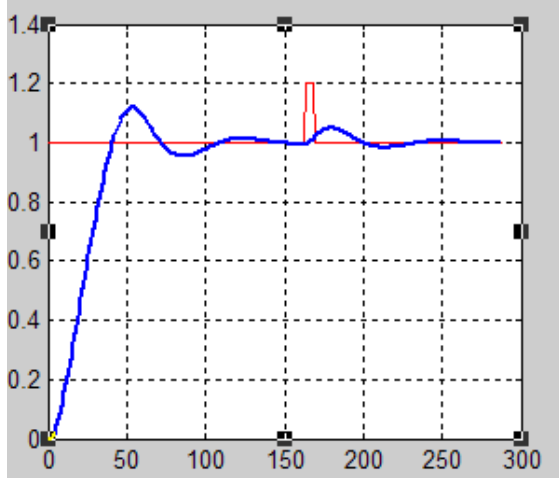

Fig.4 The Anti-interference of PID

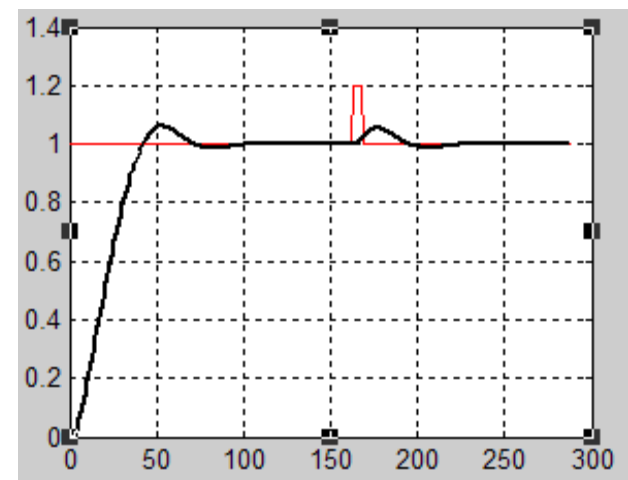

Fig.5 The Anti-interference of PIDNN

\section{Conclusion}

PID neural networks is neither a simple superposition of the neural network and conventional PID, nor the method of neural network to adjust the parameter values of PID. It is to build the controller model neural network model according to According to the neural network rules, and using mature experience of PID controller to select the initial connection weights so that PID neural networks controller not only have the advantages of strong self-learning ability of neural network, good dynamic and static performance, Strong robustness, but also retains the properties that control system is equivalent to the PID controller in the initial operating state. Although the results of computer simulation is used for PID neural networks,but it does not depend on the exact model of the controlled object, and can ensure the stability of the initial state of the control system, it also can be able to replace the PID control technology and be suitable for the actual application of complex control systems.

In the paper simulation of unit step response and Anti-interference response to the barrel temperature control system were carried out in MATLAB environment. The simulation results show that temperature control system based on the PID neural networks the strong ability to adapt working conditions and inhibit interference, and can effectively overcome the characteristics of nonlinear, time-variant and large delay-time to achieve better control effect.

\section{References}

[1] Lin Rongchuan. The Temperature Control and Interference of Injection Molding Machine [J]. Chinese plastics, 2011,25 (3): 89-93.

[2] Shuhuai Lin .PID Neural Networks and its Control System [M]. Beijing: National Defense Industry Press, 2006.

[3] Li Minghui, Li Zhengqi. Decoupling Control Strategy in Barrel Temperature of Injection Molding Machine based on Neural Networks [J]. Ceramics, 2012,(4):17-19.

[4] Zhong Hanru. Control System of Injection Molding Machine [M].Beijing: Chemical Industry Press, 2004.

[5] Wang Huiqing, Gu Shuping.The Control System Design for Non-automated Barrel Temperature of Injection Molding Machine. Techniques of Automation and Applications, 2009,28 (3): 116-118. 